\title{
The Fraunhofer Pattern of a Wave Field Generating by a System of Coherent Emitting Point Sources
}

\author{
A.Zh. Khachatrian \\ National Polytechnic University of Armenia, 105 Teryan Street, Yerevan, 0009, Armenia \\ e-mail: ashot.khachatrian@gmail.com
}

Received: December 3, 2021; Revised: December 20, 2021; Accepted: December 29, 2021

\begin{abstract}
The problem of description of a wave field generating by a system of coherent point sources is considered. This problem is discussed for two well-known approximations, the Fresnel and Fraunhofer patterns, correspondingly. It is shown, that for validity of the both approximations the problem has to contain two dimensionless small parameters. First of them is the wave parameter of the sources area and the second one is the wave parameter corresponding to the observation area. In the framework of the Fraunhofer pattern the Laue conditions of diffraction maximums for a system of periodic arranged sources is derived.
\end{abstract}

Keywords: superposition field, system of many sources, diffraction, Fresnel and Fraunhofer patterns

https://doi.org/10.54503/18291171-2021.14.4-201

\section{Introduction}

In the paper we consider the problem of description of a scalar wave field (or a component of some vector filed) generating by the system of coherent emitting point sources. This problem has an important role in the wave theory and lies in the base of calculations of the working parameters for many optic system and phase lattices [1-7]. It is known that when the wave process excited by the sources is very weakly scattered or acted on them, then the superposition field is given by the following sum:

$$
U(\vec{R}, t)=\sum_{j=1}^{N} \frac{a_{0}}{L_{j}} \cos \left[\omega t-k L_{j}+\gamma_{j}\right],
$$

where $N$ is a number of the point sources, $a_{0}$ is the sphere wave amplitude and the space vector $\vec{R}$ shows the observation point. Here $L_{j}$ is the distance between the observation point and the $j$ source. The quantities $\omega$ and $k$ are the frequency and the wave number of the generated wave field. We assume that the dispersion low $\omega(k)$ can be in any form, for example, has a linear form:

$$
\omega(k)=c k,
$$

where $c$ is the propagation velocity of waves.

In the expressions of the space dependent parts of phases of sphere waves (see (1)), i.e. for the expressions

$$
\varphi_{j}(\vec{R})=k L_{j}-\gamma_{j} \quad(j=1,2, \cdots, N)
$$


we took into account that the wave field generation by the different sources can differ by the phases at the initial time moment. So, the quantities $\gamma_{j}$ are the initial magnitudes of the phases.

In general, the study of expression (1) is rather difficult, except the case when $N=2$, which corresponds to the well-known case of interference or the case of a diffraction grating, when $N>>1$ the emitters are located on the same straight line $[8,9]$. However, if we restrict ourselves to considering the wave field only at certain points and at the same time impose certain conditions on the parameters of the problem, then, as is known, one can come to a more simplified form of writing the sum (1) that preserves the main features of diffraction (see, for example, [10-15]).

In the framework of this paper we discuss the problem of description of the superposition field (1). In particular, we consider the problem of the determination of the space positions of the points corresponded to the maximum and minimum of the intensity of the wave field (1). It is clear that the position of these points is directly related to the spatial location of the emitters. In general, the presented work is devoted to the study of the expression of the superposition field (1) and its consideration in the so-called pictures of Fresnel and Fraunhofer. Below we consider the problem in general terms, as possible, and here the main emphasis is on the initial consideration of the fields of single sources in the corresponding figures, i.e., in a highly simplified form.

\section{The problem statement}

Let the source system be located near some point $O$, which we will call the center of the source area. Let us also assume that the observations are carried out within a certain area and choose a point $O^{\prime}$ inside the observation area. We will call this point as a central observation point. The straight line drawn through the points $O O^{\prime}$ we call as a main observation axis and the distance between the points $O$ and $O^{\prime}$ as an average light of observation. Below for description of the wave field we will associate with central point of the source area $O$ the origin of a rectangular coordinate system and direct the axis $Z$ along the main observation axes $O O^{\prime}$ (see Fig. 1). In accordance with this choice of the coordinate system the plane $(X, Y)$ will define the across directions of observation and the axis $Z$ will define the main observation direction.

Let as denote by $\vec{r}_{j}(j=1,2, \cdots, N)$ the space vectors showing the positions of the sources;

$$
\vec{r}_{j}=x_{j} \cdot \vec{e}_{x}+y_{j} \cdot \vec{e}_{y}+z_{j} \cdot \vec{e}_{z}
$$

where $x_{j}, y_{j}, z_{j}$ are the corresponding coordinates of the $j$ - th source and $\vec{e}_{x}, \vec{e}_{y}, \vec{e}_{z}$ are the unit dimensionless vectors showing the positive directions of the axis $X, Y, Z$. In accordance with (4) (see Fig. 1 as well) the vector $\vec{R}_{j}$ directed from the location point of the $j$ - th source to the observation point can be presented as;

$$
\vec{R}_{j}=\left(x-x_{j}\right) \cdot \vec{e}_{x}+\left(y-y_{j}\right) \cdot \vec{e}_{y}+\left(z-z_{j}\right) \cdot \vec{e}_{z}
$$

where $x, y, z$ are the coordinates of an observation point;

$$
\vec{R}=x \cdot \vec{e}_{x}+y \cdot \vec{e}_{y}+z \cdot \vec{e}_{z}
$$

Below we investigate the wave field pattern in the plane perpendicular to the main observation direction $Z$ (see Fig. 1). Let the observation plate crosses the $Z$ in the point $Z$. Then, the observation point in the observation plane will be defined by the vector $\vec{\rho}$; 


$$
\vec{R}=\vec{\rho}+z \cdot \vec{e}_{z}
$$

and

$$
\vec{\rho}=x \cdot \vec{e}_{x}+y \cdot \vec{e}_{y}
$$

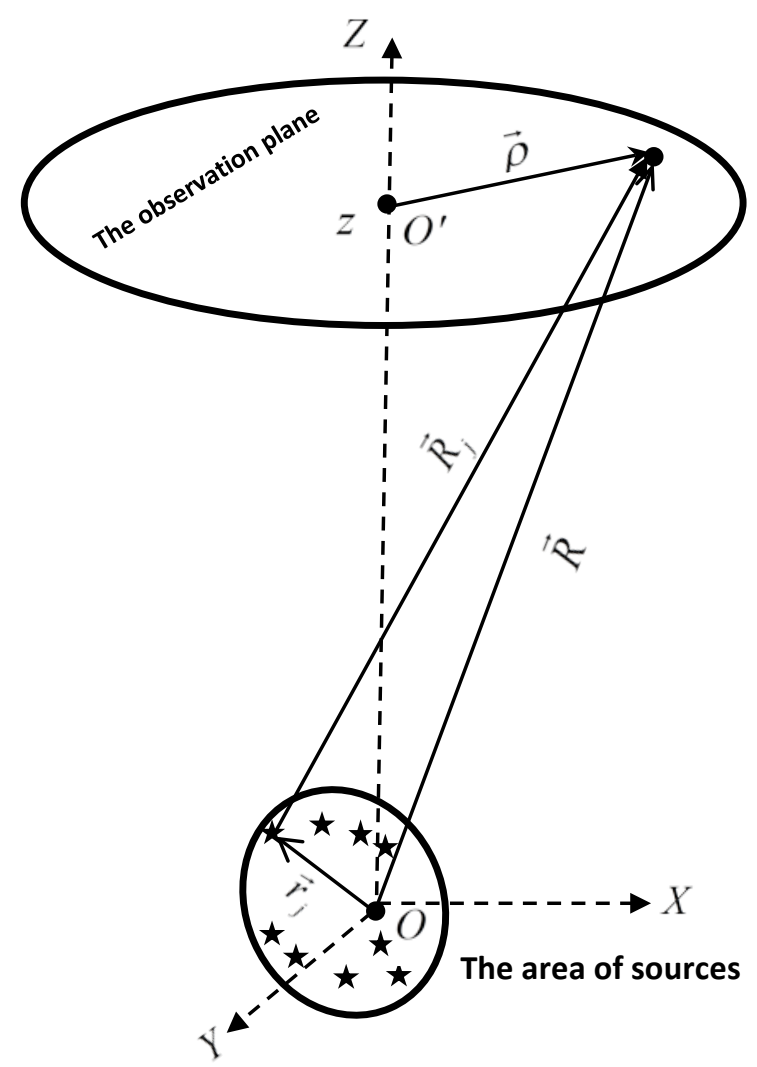

Fig. 1. The diffraction experiment scheme, where the source area and the observation area are indicated, as well as the vectors necessary for the description are introduced.

Using (5) - (6) for the distance $L_{j}$ between the observation point and the $j$-th source (see (1)) one can write:

$$
L_{j}=\left|\vec{R}_{j}\right|=\sqrt{\left(z-z_{j}\right)^{2}+\left(x-x_{j}\right)^{2}+\left(y-y_{j}\right)^{2}} .
$$

Let us introduce the positive quantities $d_{\square}, d_{\perp}$ and $\rho$ which determine the linear sizes of the sources and observation areas, correspondingly. The parameter $d_{\square}$ will limit the magnitudes of the longitudinal size of the source area;

$$
\left|z_{j}\right| \leq d_{\square}(j=1,2, \cdots, N)
$$

and the parameters $\rho_{\perp}, d_{\perp}$ limit the magnitudes of the shear sizes of the problem;

$$
|x| \leq \rho,|y| \leq \rho \text { and }\left|x_{j}\right| \leq d_{\perp},\left|y_{j}\right| \leq d_{\perp}(j=1,2, \cdots, N) .
$$

Now, by using (8) let us consider the amplitude part of a one sphere wave (see (1)); 


$$
\frac{a_{0}}{L_{j}}=\frac{a_{0}}{\sqrt{\left(z-z_{j}\right)^{2}+\left(x-x_{j}\right)^{2}+\left(y-y_{j}\right)^{2}}} .
$$

If the experimental conditions are such that the linear sizes of the both areas much less than the average distance of observation $L$;

i.e. when

$$
L=|z|
$$

$$
d_{\square}<<L, d_{\perp}<<\text { and } \rho<<L,
$$

then all amplitudes of the sphere waves can be consider as equal to each other $[8,9]$;

$$
\frac{a_{0}}{L_{j}} \approx \frac{a_{0}}{L}
$$

This equality means, that under the conditions (13) when the across and longitudinal sizes of the source region and the observation area are much less than the average observation distance then the amplitudes of the waves arriving at the observation point from different sources can be considered equal to each other. At the same time the conditions (13) are not sufficient conditions to do corresponding simplifications in expressions of the wave phases $\varphi_{j}(\vec{R})$. Indeed, the phase expression (3) appears in the argument of the oscillatory function, in this case in the cosine argument. It means that when the parameters of the problem change, then the phase change should be insignificant (see below).

\section{The phase of a sphere wave in the Fresnel and Fraunhofer picture}

As it was shown the paragraph 2 for a system of many point sources the problem of determination of the maximums and minimums of the superposition field intensity is reduced to investigation of the path differences for all possible pairs of sources. In the general case of sources with an arbitrary space location the consideration faces many difficulties. At the same time, when certain conditions are imposed on the parameters of the problem, the expressions for the sphere wave phases, in particular, their dependence on the spatial coordinates, are simplified.

Now taking into account the conditions (13) (see (9), (10) and (12), as well);

$$
|x|,|y|,\left|x_{j}\right|,\left|y_{j}\right|,\left|z_{j}\right|<<=|z|,
$$

we consider the question of the possibility of simplifying the phase (3) of the wave field of a point source, namely, its spatially dependent part (see (8));

$$
\varphi_{j}(\vec{R})=\psi_{j}(\vec{R})-\gamma_{j}, \quad \psi_{j}(\vec{R}) \equiv \psi\left(\vec{R}, \vec{r}_{j}\right)
$$

and

$$
\psi\left(\vec{R}, \vec{r}_{j}\right)=k L_{j}=k \sqrt{\left(z-z_{j}\right)^{2}+\left(x-x_{j}\right)^{2}+\left(y-y_{j}\right)^{2}} .
$$

Let us write down this expression in the form of:

$$
\psi\left(\vec{R}, \vec{r}_{j}\right)=k\left|z-z_{j}\right| \sqrt{1+\Delta_{j}},
$$


where

$$
\Delta_{j}=\frac{\left(x-x_{j}\right)^{2}+\left(y-y_{j}\right)^{2}}{\left(z-z_{j}\right)^{2}} .
$$

As it seen from the conditions (15):

$$
\Delta_{j}<<1
$$

Expanding the radical of (18) into a Taylor series in powers of $\Delta_{j}$ one gets:

$$
\psi\left(\vec{R}, \vec{r}_{j}\right)=k\left|z-z_{j}\right|\left(1+\frac{\Delta_{j}}{2}-\frac{\Delta_{j}^{2}}{8}+\frac{\Delta_{j}^{3}}{16}-\cdots\right) .
$$

Using (19) for (21) it can be written:

$$
\psi\left(\vec{R}, \vec{r}_{j}\right)=k\left|z-z_{j}\right|+\frac{\sigma_{j}}{2}-\frac{1}{k\left|z-z_{j}\right|} \frac{\sigma_{j}^{2}}{8}+\frac{1}{\left(k\left|z-z_{j}\right|\right)^{2}} \frac{\sigma_{j}^{3}}{16}-\cdots
$$

where

$$
\sigma_{j}=k\left|z-z_{j}\right| \cdot \Delta_{j}=\frac{\left(x-x_{j}\right)^{2}+\left(y-y_{j}\right)^{2}}{\left|z-z_{j}\right|} k
$$

Since we have considered that $\left|z_{j}\right|<<|z|$ (see (15)) for (22), (23) it can be found

$$
\psi\left(\vec{R}, \vec{r}_{j}\right)=k\left(L-z_{j}\right)+\frac{\sigma_{j}}{2}-\frac{1}{k \cdot L} \frac{\sigma_{j}^{2}}{8}+\frac{1}{(k \cdot L)^{2}} \frac{\sigma_{j}^{3}}{16}-\cdots \quad(z>0)
$$

where

$$
\sigma_{j}=\frac{\left(x-x_{j}\right)^{2}+\left(y-y_{j}\right)^{2}}{L} k \text {. }
$$

Below we will assume that in (24) the ratio $1 /(k \cdot L)<<1$, which will mean that

$$
\lambda / L<<1 \text {, }
$$

where $\lambda$ is a wave length.

In the diffraction theory the parameter $\sigma_{j}$ is known as the wave parameter (see, for example, [9]). If $\sigma_{j} \square 2 \pi$ when for the series (24) the following expression can be applied:

$$
\psi\left(\vec{R}, \vec{r}_{j}\right)=k\left(L-z_{j}\right)+\frac{\sigma_{j}}{2}-\frac{1}{k \cdot L} \frac{\sigma_{j}^{2}}{8} .
$$

The given expression is the sphere wave phase written for the Fresnel picture. If

$$
\sigma_{j}<<2 \pi
$$

from (24) one gets: 


$$
\psi\left(\vec{R}, \vec{r}_{j}\right)=k\left(L-z_{j}\right)+\frac{\sigma_{j}}{2}
$$

which is the expression of the sphere phase written for the Fraunhofer picture.

Using (25) the last expression can be presented as well:

$$
\psi\left(\vec{R}, \vec{r}_{j}\right)=k\left(L-z_{j}\right)+\frac{\left[x^{2}+y^{2}+x_{j}^{2}+y_{j}^{2}\right]-2\left[x \cdot x_{j}+y \cdot y_{j}\right]}{2 L} k .
$$

In the case of when the source locates in the origin of the coordinate system, i.e. when we have $x_{j}=0, y_{j}=0$ and $z_{j}=0$, it is easy to see that expression (30) takes the form of:

$$
\psi(\vec{R}, 0) \equiv \psi_{0}=k \cdot L+k \frac{x^{2}+y^{2}}{2 L} .
$$

Let us introduce the quantity

$$
\Delta_{j}=k \cdot z_{j}-\frac{\left[x_{j}^{2}+y_{j}^{2}\right]-2\left[x \cdot x_{j}+y \cdot y_{j}\right]}{2 L} k .
$$

When the phase (30) can be written as:

$$
\psi\left(\vec{R}, \vec{r}_{j}\right)=\psi_{0}-\Delta_{j}
$$

Note that when $x_{j}=0, y_{j}=0$ and $z_{j}=0$ the quantity $\Delta_{j}$ takes zero value:

$$
\Delta_{j} \equiv \Delta\left(\vec{R}, \vec{r}_{j}\right) \text { and } \Delta(\vec{R}, 0)=0
$$

The last means that the value of the quantity $\Delta_{j}$ shows the change of the sphere wave phase in a point of a radius vector $\vec{R}$ when the source is moved from the coordinate system origin to a point of a radius vector $\vec{r}_{j}$.

First of all let us note that by using the formula (25) the condition (28) providing the observation of the Fraunhofer picture can be transformed into the following conditions:

$$
\frac{x^{2}}{L} k<2 \pi, \frac{y^{2}}{L} k<<2 \pi \text { and } \frac{x_{j}^{2}}{L} k<<2 \pi, \frac{y_{j}^{2}}{L} k<<2 \pi .
$$

From these conditions it follows that:

$$
\frac{x \cdot x_{j}}{2 L} k<<2 \pi \text { and } \frac{y \cdot y_{j}}{2 L} k<<2 \pi
$$

as well.

In accordance with the above results the wave field (1) generated by a system of point sources in the Fraunhofer picture is given by the following expression;

$$
U(\vec{R}, t)=\frac{a_{0}}{L} \sum_{j=1}^{N} \cos \left[\omega t-\psi_{0}-\Delta_{j}-\gamma_{j}\right](z>0),
$$


It is easy to see that for any space point by means of the shift of the time origin calculation $\left(\Delta t=\psi_{0} / \omega\right)$ this sum is reduced to:

$$
U(\vec{R}, t)=\frac{a_{0}}{L} \sum_{j=1}^{N} \cos \left[\omega t-k \cdot z_{j}+\frac{\left[x_{j}^{2}+y_{j}^{2}\right]-2\left[x \cdot x_{j}+y \cdot y_{j}\right]}{2 L} k-\gamma_{j}\right] .
$$

As it follows from this expression the character of the superposition field depends on the shear coordinates of the observation point $x, y$ linearly. This dependence as a function of the shear coordinates of the sources $x_{j}, y_{j}$ also has a quadratic term.

Using the connection $k=2 \pi / \lambda$ and the notations (10) one can present the conditions of the Fraunhofer picture (35) as:

$$
\frac{\rho^{2}}{\lambda L}<<1 \text { and } \frac{d_{\perp}^{2}}{\lambda L}
$$

Taking into account the conditions (see (10) and (15));

$$
\rho / L<<1 \text { and } d_{\perp} / L<<1 \text {, }
$$

it can be seen that the consideration of the conditions (39) is reduced to investigation of the values of the following fractions:

$$
\rho / \lambda \text { and } d_{\perp} / \lambda
$$

Note it is assumed that $\lambda / L<<1$ as well (see (26)).

On the base of (39)-(41) it can be distinguished the following four situations, when for the superposition field the Fraunhofer approximation can be applied:

$$
\begin{aligned}
& \lambda<<\rho, \lambda<<d_{\perp} ; \\
& \lambda<<\rho, \lambda \square d_{\perp} ; \\
& \lambda \square \rho, \lambda<<d_{\perp} ; \\
& \lambda \square \rho, \lambda \square d_{\perp} .
\end{aligned}
$$

It is clear that when

$$
\lambda>>\rho \text { or } \lambda>d_{\perp},
$$

then the wave field description should be done be means of a higher approximation, for example, by means of the Fresnel approximation.

In the usual problems of the interference and diffraction we observe the situations which correspond to the cases of (42), (43). So, for the interference problems the values of the parameters are approximately as follows:

$$
\lambda \square 10^{-7} \mathrm{~m}, \rho \square 10^{-3} \mathrm{~m}, d_{\perp} \square 10^{-3} \mathrm{~m} .
$$

In the case of the optic diffraction gratings:

$$
\lambda \square 10^{-7} \mathrm{~m}, \rho \square 10^{-3} \mathrm{~m}, d_{\perp} \square 10^{-6} \mathrm{~m} .
$$

Note that in experiments on optical diffraction, in addition to condition (44), we also have: 


$$
d_{\perp}<<\rho \text {. }
$$

The last means that in the expression of the superposition field one can take:

$$
\left|x_{j}\right|,\left|y_{j}\right|<<|x|,|y|
$$

and consider the wave field in the form of:

$$
U(\vec{R}, t)=\frac{a_{0}}{L} \sum_{j=1}^{N} \cos \left[\omega t-k \cdot z_{j}-\frac{x \cdot x_{j}+y \cdot y_{j}}{L} k-\gamma_{j}\right] .
$$

As we will show below, by mean of the wave field presentation (49) many well-known results of diffraction theory for periodic structure can be easily derived.

It is interesting to show the transition of the expression (38) into (49), where the superposition field dependence on the coordinates of the sources has a linear character. Applying the well-known trigonometric relation about the cosine of the sum of two angles to expression (38) we obtain:

$$
\begin{aligned}
U(\vec{R}, t) & =\frac{a_{0}}{L} \sum_{j=1}^{N} \cos \left[\frac{x_{j}^{2}+y_{j}^{2}}{2 L} k\right] \cos \left[\omega t-k \cdot z_{j}-\frac{x \cdot x_{j}+y \cdot y_{j}}{L} k\right]- \\
& -\frac{a_{0}}{L} \sum_{j=1}^{N} \sin \left[\frac{x_{j}^{2}+y_{j}^{2}}{2 L} k\right] \sin \left[\omega t-k \cdot z_{j}-\frac{x \cdot x_{j}+y \cdot y_{j}}{L} k\right] .
\end{aligned}
$$

Since

$$
\frac{x_{p}^{2}+y_{p}^{2}}{2 L} k \geq 0,
$$

and in accordance with (35) this ratio is a small one can take:

$$
\cos \left[\frac{x_{p}^{2}}{2 L} k\right] \approx 1 \text { and } \sin \left[\frac{x_{p}^{2}}{2 L} k\right] \approx 0 .
$$

Using (51) it is easy to see that the sum (50) takes the form of (49).

\section{The wave field generated by the system of periodic arranged sources}

In this section we will consider the problem of description of the wave field generated by the system of periodic arranged sources. Below we consider the sum (49), when the coordinates of sources will correspond to nodes of some regular lattice. In the case of a three-dimensional structure, the locations of the sources are conveniently indicated by means of three indexes, rather than one index. Describing the emitting structure by mean of three indexes the wave field (49) can be presented in the form of:

$$
U(\vec{R}, t)=\frac{a_{0}}{L} \sum_{h=1}^{N_{x}} \sum_{p=1}^{N_{y}} \sum_{l=1}^{N_{z}} \cos \left[\omega t+\Delta_{h p l}\right]
$$

where 


$$
\Delta_{h p l}=-k \cdot z_{l}-\frac{x \cdot x_{h}+y \cdot y_{p}}{L} k-\gamma_{h p l} .
$$

It is clear that the number of sources of such structure $N=N_{x} N_{y} N_{z}$.

As it was mentions above the quantities $\gamma_{j}\left(\gamma_{h p l}\right)$ are the initial magnitudes of the phases $\varphi_{j}(\vec{R})=k L_{j}-\gamma_{j}$ (see (3)). Let the radiation of sources are induced by the presence of some primary field, which will define the magnitudes of the quantities $\gamma_{h p l}$. This field is an external mechanism for creating forces for all sources, under the action of which the sources begin to generate waves. It is clear that, if we neglect the influence of the secondary field created by the sources on the sources themselves, then the oscillations of the sources will be determined only by the magnitude of the primary field at the points of the sources and its polarization. For example, in the diffraction of X-rays on a crystal lattice, when the primary X-ray penetrating into the volume of the crystal, leads to the excitation of secondary waves on the atoms of the lattice. As is known, in this case, the difference in wave paths from different atoms is determined not only by their mutual position, but also by the directionality of the primary beam [10-15].

Let the primary field has the form of a harmonic plane wave;

$$
\Phi(\vec{r}, t)=\Phi_{0} \cos (\omega t-\vec{K} \vec{R})
$$

where $\Phi_{0}$ is the amplitude of the primary wave and $\vec{K}$ its wave vector. Note that the modules of the waves vectors $\vec{K}$ and $\vec{k}$ (see (2)) should be equal to each other:

$$
K=k \text {. }
$$

Since the time delays associated with the processes of absorption and re-emission of the energy of the external field for all sources are the same, we will write the initial phases $\gamma_{j}$ as:

or

$$
\gamma_{j}=\vec{K} \cdot \vec{r}_{j}
$$

$$
\gamma_{h p l}=K_{x} \cdot x_{h}+K_{y} \cdot y_{l}+K_{z} \cdot z_{p} \cdot
$$

Using (57) for (53) one can write:

$$
\begin{aligned}
& \Delta_{h p l}=-q_{x} x_{h}-q_{y} y_{p}-q_{z} \cdot z_{l}, \\
& q_{x}=\frac{x}{L} k+K_{x}, q_{y}=\frac{y}{L} k+K_{y}, q_{z}=\left(k+K_{z}\right) .
\end{aligned}
$$

Let us consider the intensity of the superposition field;

$$
I=\frac{1}{T} \int_{0}^{T} U^{2}(\vec{R}, t) d t
$$

Using (52) one can get:

$$
I=\frac{1}{2}\left(\frac{a_{0}}{L}\right)^{2} \sum_{h=1}^{N_{x}} \sum_{h^{\prime}=1}^{N_{x}} \sum_{l=1}^{N_{y}} \sum_{l^{\prime}=1}^{N_{y}} \sum_{p=1}^{N_{z}} \sum_{p^{\prime}=1}^{N_{z}} \cos \left(\Delta_{h p l}-\Delta_{h^{\prime} p^{\prime} l^{\prime}}\right) .
$$


As it should be the intensity depends on the phase differences $\Delta_{h p l}-\Delta_{h^{\prime} p^{\prime} l^{\prime}}$ between the waves emitted by sources. Note that the number of members in the sum (61) equals to $N_{x}^{2} N_{y}^{2} N_{z}^{2}$. As it follows from this formula if

$$
\Delta_{h p l}-\Delta_{h^{\prime} p^{\prime} l^{\prime}}=2 \pi m_{j},\left(m_{j}=0, \pm 1, \pm 2, \cdots\right)
$$

then the wave the wave filed oscillates with ta maximum value;

$$
I_{\max }=\left(\frac{a_{0}}{L}\right)^{2} N_{x}^{2} N_{y}^{2} N_{z}^{2}
$$

For a periodic structure, when the coordinates of sources can be presented in the form of

$$
x_{h}=x_{1}+a \cdot(h-1), y_{l}=y_{1}+b \cdot(l-1), z_{p}=z_{1}+c \cdot(p-1),
$$

the phase differences can be written:

$$
\Delta_{h p l}-\Delta_{h^{\prime} p^{\prime} l^{\prime}}=a\left(h^{\prime}-h\right) q_{x}+b\left(l^{\prime}-l\right) q_{y}+c\left(p^{\prime}-p\right) q_{z}
$$

In this case the sum (61) can be calculated:

$$
I=\frac{1}{2}\left(\frac{a_{0}}{L}\right)^{2} \frac{\sin ^{2}\left[\frac{q_{x} a \cdot N_{x}}{2}\right]}{\sin ^{2}\left[\frac{q_{x} a}{2}\right]} \frac{\sin ^{2}\left[\frac{q_{y} b \cdot N_{y}}{2}\right]}{\sin ^{2}\left[\frac{q_{y} b}{2}\right]} \frac{\sin ^{2}\left[\frac{q_{z} c \cdot N_{z}}{2}\right]}{\sin ^{2}\left[\frac{q_{z} c}{2}\right]} .
$$

As it is seen from this result the intensity takes the maximum, when

$$
q_{x} a=2 \pi n_{x}, q_{y} b=2 \pi n_{y}, q_{z} c=2 \pi n_{z},
$$

where $n_{x}, n_{y}, n_{z}=0, \pm 1, \pm 2 \cdots$ and the maximum value is (63). In fact, the obtained formulas (67) are the well-known Laue conditions, which determine the diffraction maximums of X-rays scattered on the crystal lattice (see, for example, $[16,17]$ ).

Let us introduce the quantities $\beta_{x}, \beta_{y}, \beta_{z}$, which are the angles between the vector $\vec{K}$ (see (55)) and the $X, Y, Z$ axis;

$$
\vec{K}=k\left(\cos \left[\beta_{x}\right] \cdot \vec{e}_{x}+\cos \left[\beta_{y}\right] \cdot \vec{e}_{y}+\cos \left[\beta_{z}\right] \cdot \vec{e}_{z}\right) .
$$

Since $K_{x}=k \cos \left[\beta_{x}\right], K_{y}=k \cos \left[\beta_{y}\right], K_{z}=k \cos \left[\beta_{z}\right]$ the conditions (67) can be written in the form of:

$$
\begin{aligned}
& \frac{x}{L}+\cos \left[\beta_{x}\right]=\frac{n_{x} a}{\lambda}, \\
& \frac{y}{L}+\cos \left[\beta_{y}\right]=\frac{n_{y} b}{\lambda},
\end{aligned}
$$




$$
\left(1+\cos \left[\beta_{z}\right]\right)=\frac{n_{z} c}{\lambda}
$$

It is important to note that the angles $\beta_{x}, \beta_{y}, \beta_{z}$ defining the direction of the incident wave are not independent from each other parameters, as it can appear at first glance. Indeed in the cylindrical coordinate system the vector $\vec{K}$ can be presented as:

$$
\vec{K}=k\left(\sin [\beta] \cdot \cos [\varphi] \cdot \vec{e}_{x}+\sin [\beta] \cdot \sin [\varphi] \cdot \vec{e}_{y}+\cos [\beta] \cdot \vec{e}_{z}\right),
$$

where $\beta_{z} \equiv \beta$ is the angle of incident and $\varphi$ is the polar angle in the $X, Y$ plane; $\cos \left[\beta_{x}\right]=\sin [\beta] \cdot \cos [\varphi], \quad \cos \left[\beta_{y}\right]=\sin [\beta] \cdot \sin [\varphi]$. Using (72) the conditions (69)-(71) can be presented as:

$$
\begin{aligned}
& \frac{x}{L}+\sin [\beta] \cdot \cos [\varphi]=\frac{n_{x} \lambda}{a}, \\
& \frac{y}{L}+\sin [\beta] \cdot \sin [\varphi]=\frac{n_{y} \lambda}{b}, \\
& (1+\cos [\beta])=\frac{n_{z} \lambda}{c} .
\end{aligned}
$$

These formulas are the conditions defining the locations of maximums of a wave field generated by a system of periodic arranged sources.

It is visible, that the obtained result includes the solutions of many well-known diffraction problems. So, in the case of normal incident $\beta=0$ from (73)- (75) one gets:

$$
x=n_{x} \lambda L / a, y=n_{y} \lambda L / b, n_{z} \lambda=2 c .
$$

The first and second equations correspond to the maximum condition of for the one dimensional diffraction and the last equation corresponds to the well-known Wolf-Bragg formula.

\section{Conclusion}

Within the framework of this work, the problem of description the wave field created by a system of point sources was considered. We have shown that for a simplified description of the field in the Fresnel or Fraunhofer pictures, in addition to the conditions for the smallness of the source region and the remoteness of the observation region, it is also necessary to have two small parameters. First of them is the wave parameter of the sources area and the second one is the wave parameter corresponding to the observation area. We also obtained the expression of the superposition field for a three dimensional periodic system. On the base of the developed approach we derived the well-known Laue conditions determining the diffraction maximums of X-rays scattered on the crystal lattice.

\section{Referenses}

[1] D. Hebri, S. Rasouli, A. M. Dezfouli, J. Opt. Soc. Am. A 36 (2019) 839.

[2] N. Morimoto, S. Fujino, Y. Ito, A. Yamazaki, I. Sano, T. Hosoi, H. Watanabe, T. Shimura, Optics Express 23 (2015) 29399.

[3] B. Xie, X. Cai, Z.H. Xiao, Optics Communications, 285 (2012) 133.

[4] S. Rasouli, F. Sakha, M. Yeganeh, Meas. Sci. Technol. 29 (2018) 085201. 
[5] A.Naqavi, H. P. Herzig, M. Rossi, J. Opt. Soc. Am. B 33 (2016) 2374.

[6] V. Saveljev, S. Kim, H. Lee, H Kim, B. Lee, Optics Express 24 (2016) 2905.

[7] H. Pang, J. Wang, M. Zhang, A. Cao, L. Shi, Q. Deng, Optics Express 25 (2017) 14323.

[8] G.S. Gorelik, Oscillations and Waves (Fizmatlit, Moscow, 2007).

[9] M. Born, E. Wolf, Principles of Optic (Oxford University Press, New York, 2013).

[10] A.J. Dicken, J.P.O. Evans, K.D. Rogers, C. Greenwood, S.X. Godber, D. Prokopiou, N. Stone, J.G. Clement, I. Lyburn, R.M. Martin, P. Zioupos, Optics Express 23 (2015) 13443.

[11] P. Evans, K. Rogers, J. Chan, J. Rogers, A. Dicken, Appl. Phys. Lett. 97 (2010) 204101.

[12] E. Gregoryanz, O. Degtyareva, M. Somayazulu, R.J. Hemley, H.-K. Mao, Phys. Rev. Lett. 94, (2005) 185502.

[13] M. Kagias, Z. Wang, P. Villanueva-Perez, K. Jefimovs, M. Stampanoni, Phys. Rev. Lett. 116 (2016) 093902.

[14] M. Wieczorek, F. Schaff, C. Jud, D. Pfeiffer, F. Pfeiffer, T. Lasser, Sci. Rep. 8 (2018) 14345.

[15] J. Wang, F. Coppari, R.F. Smith, J.H. Eggert, A.E. Lazicki, D.E. Fratanduono, J.R. Rygg, T.R. Boehly, G.W. Collins, T.S. Duffy, Phys. Rev. B 94 (2016) 104102.

[16] H.J. Pain, The Physics of Vibrations and Waves (John Wiley \& Sons, Ltd, London, 1976).

[17] Ch. Kittel, Introduction to the Solid State Physics. The Physics of Vibrations and Waves (John Wiley \& Sons, Ltd, London, 1974). 